\title{
Biodegradable Polymer-Coated, Gelatin Hydrogel/Bioceramics Ternary Composites for Antitubercular Drug Delivery and Tissue Regeneration
}

\author{
Mintao Xue, Hongtao Hu, Yuanquan Jiang, Jichun Liu, Hailong He, and Xiaojian Ye \\ Department of Orthopedics, Changzheng Hospital of Second Military Medical University, Shanghai 200003, China
}

Correspondence should be addressed to Hailong He, hailong0530@126.com and Xiaojian Ye, yexj2002@163.com

Received 20 February 2012; Accepted 28 March 2012

Academic Editor: Sevan P. Davtyan

Copyright () 2012 Mintao Xue et al. This is an open access article distributed under the Creative Commons Attribution License, which permits unrestricted use, distribution, and reproduction in any medium, provided the original work is properly cited.

\begin{abstract}
A simple and effective strategy for the treatment of osteoarticular tuberculosis is proposed through combining tissue engineering approach with anti-tuberculosis drug therapy. A series of tricalcium phosphate bioceramics (TPB) composites, coated by degradable polymer outside and loaded with rifampicin (RFP)-containing gelatin hydrogel inside, were thus fabricated and successfully applied to deliver antitubercular drug RFP into osseous lesion and concomitantly to induce tissue regeneration. RFP-loaded gelatin hydrogel/TPB composites could be readily prepared by filling RFP-containing gelatin solution into TPB and then in situ crosslinking of gelatin with calcium ions. Depending on the concentrations of RFP, the loading efficiency of RFP in the composites varied in the range from approximately $2 \%$ to $5 \%$. Moreover, the surface of these binary composites could be further coated by a biodegradable polymer, yielding biodegradable polymer-coated, RFP-containing gelatin hydrogel/TPB ternary composites. It was shown that in vitro release of RFP from the ternary composites could be effectively sustained for a long period of time. Besides, these composites revealed good biocompatibility towards the survival of MC-3T3 cells in vitro and could be used for tissue regeneration in vivo in a rabbit model. The results indicate that TPB ternary composites have great potential for the treatment of osteoarticular tuberculosis.
\end{abstract}

\section{Introduction}

In the past two decades, much effort has been directed towards the development of antitubercular drugs for tuberculosis therapy [1]. Although a few of them have been proven to be very efficacious for clinical treatment of tuberculosis, the advancement of tolerance by the tubercle bacillus may be a massive hurdle. To address this issue, combined therapy using multiple drugs such as rifampicin (RFP) and isoniazid (INH) has been studied widely [2]. By this approach, a relatively long-term administration (e.g., 6-9 months) is, however, normally required for the patients, becoming a huge burden to the patients. Besides, an undesirable side effect from these drugs is another concern. For example, RFP, one of the first line antitubercular drugs, is known to cause serious liver damage $[3,4]$. Recently, the researches on controlled release of antitubercular drugs have received much attention. The concept involves the utility of the drug delivery system that can carry antitubercular drugs and mediate long-term, sustained release of the drugs. This kind of formulation gains a few advantages: (1) the administration frequency can be reduced owing to sustained drug release; (2) the dose can be used at a reduced level as compared to that used in traditional oral administration; (3) side effects such as a liver damage can be weaken due to a low drug concentration in the body. Furthermore, different antitubercular drugs can be administrated together from the delivery system, giving rise to a sustainable delivery for combined drug therapy. For these reasons, a few drug delivery systems from biocompatible, nontoxic polymers have been developed recently $[5,6]$. For example, Onoshita et al. reported on PLGA microspheres serving as delivery systems for controlled release of RFP within 10 days [7]. More recently, Zhu et al. prepared mesoporous silica nanoparticles for combined release of RFP and INH and a relatively longer release term of 20-30 days could be obtained 
[8]. However, the knowledge on in vivo biocompatibility profile of mesoporous silica nanoparticles is limited. It has been suggested that the cytotoxicity of mesoporous silica nanoparticles is dose-dependant [9].

Tuberculous arthritis is an extrapulmonary complication of tuberculosis [10]. The number of the patient suffering from this form of arthritis accounts for 1-3\% of all tuberculosis cases. Because the osseous lesion developed by tubercle bacillus normally has poor blood supply, it is hard to achieve high efficacy for the patients when they are administrated intravenously with antitubercular drugs. Moreover, the patients usually have to undergo the debridement operation, leaving a residual cavity. It is also difficult to completely eliminate the tubercle bacillus survived in the cavity via traditional systematic drug therapy. Importantly, the cavity needs to be filled and closed for new tissue regeneration. Hence, it is highly desired to develop a handy and effective strategy to sterilize tubercle bacillus in osseous lesion and meanwhile to guild regeneration of defect tissue.

Tissue engineering has been considered as a promising strategy for regeneration of defect tissue. Normally, a biocompatible scaffold is required which allows for cell growth, differentiation, and proliferation prior to the formation of new tissue. Tricalcium phosphate bioceramics (TPB) as porous bioscaffold has been employed for tissue engineering [11]. However, TPB is highly porous and not suited for sustainable drug delivery. In this study, we aim to design an integrated TPB-based system for both tissue regeneration and osteoarticular tuberculosis therapy. To this end, a novel TPB-based composite system, coated by a biocompatible, biodegradable polymer outside and loaded with RFP-containing gelatin hydrogel inside, was prepared. RFP release in vitro under physiological conditions, in vitro cytotoxicity and in vivo biocompatibility of these composites were evaluated systematically. It was found that the TPBbased composite system could guide a long-term, sustained RFP release in vitro. Besides, this system has a trivial effect on cell viability in vitro and is suitable for tissue regeneration in a rabbit model.

\section{Experimental}

2.1. Materials. Gelatin, calcium chloride, and rifampicin were purchased from Sigma-Aldrich. Tricalcium phosphate bioceramics was ordered from Shanghai Bio-Lu Biomaterials co. Ltd. Poly(lactic acid) (PLA, $130 \mathrm{kDa}$ ), poly(lactic acid-coglycolic acid) (PLGA, $80 \mathrm{kDa}$ ), and poly (caprolactone) (PCL, $75 \mathrm{kDa})$ were prepared according to the method reported previously $[12,13]$.

\subsection{Preparation of RFP-Containing Gelatin Hydrogel/TPB} Composites. RFP-containing gelatin hydrogel/tricalcium phosphate bioceramics (TPB) binary composites were prepared by incubating TPB in the gelatin solution (PBS buffer, pH 7.4) containing RFP. Different concentrations of gelatin and RFP were applied to optimize the entrapment efficiency of RFP and the release rate of RFP. In a typical example, TPB (about $50 \mathrm{mg}, \mathrm{L} \times \mathrm{W} \times \mathrm{H}, 10 \times 5 \times 5 \mathrm{~mm}$ ) was immersed in the $10 \mathrm{~mL}$ of $1 \mathrm{wt} \%$ of gelatin solution containing RFP $(1 \mathrm{mg} / \mathrm{mL})$ for 2 days. Then, TPB was placed in a calcium chloride solution ( $5 \mathrm{wt} \%$ ) for $10 \mathrm{~min}$ for in situ crosslinking of the gelatin. Finally, the composites were collected after drying in vacuum.

\subsection{Preparation of Polymer-Coated, RFP-Loaded Gelatin} Hydrogel/TPB Composites. RFP-containing gelatin hydrogel/TPB binary composites were coated by biodegradable polymers including PLA, PLGA, and PCL, yielding polymercoated, RFP-containing gelatin hydrogel/TPB ternary composites. For example, binary composite from an RFPcontaining gelatin $(1 \mathrm{mg} R F P / \mathrm{mL})$ hydrogel and TPB was immersed in a dichloromethane solution of PLA $(1 \mathrm{mg} / \mathrm{mL})$ to allow polymer coating for about $2 \mathrm{~h}$. The composite was finally dried in vacuum to completely remove dichloromethane.

2.4. In Vitro RFP Release from Gelatin Hydrogel/TPB and Polymer-Coated Gelatin Hydrogel/TPB Composites. RFPcontaining gelatin hydrogel/TPB composites were incubated in $10 \mathrm{~mL}$ of release medium (PBS) and were gently shaken at $37^{\circ} \mathrm{C}$. The release experiment was performed in triplicates. At a different time interval, $3 \mathrm{~mL}$ of PBS was taken out and replaced with $3 \mathrm{~mL}$ of fresh PBS. The concentration of released RFP in the PBS was determined at the wavelength $470 \mathrm{~nm}$ with a Cary 50 UV-Visible Spectrophotometer (Varian). RFP concentration was calculated with a calibration curve from RFP standard solutions at different concentrations. Cumulative release was determined by normalizing the amount of RFP released at each time point with the sum of the total RFP released over the course. The entrapment efficiency (EE) of RFP was calculated by normalizing the weight of RFP loaded in the composite with that of RFP fed in the gelatin solution. The loading efficiency (LE) of RFP was calculated by normalizing the weight of RFP loaded in the composite with that of the composite alone. In vitro RFP release from biodegradable polymer-coated gelatin/TPB composites was also conducted as aforementioned method.

2.5. Scanning Electron Microscopy. The morphology of RFPcontaining gelatin/TPB composites was studied using a Hitachi S-2360 N scanning electron microscopy (SEM). The composite samples were gold sputtered and then visualized with SEM.

2.6. Toxicity Test In Vitro. To evaluate the biocompatibility of RFP-containing gelatin/TPB composites, an indirect contact method was used as reported previously [14-16]. Briefly, MC-3T3 cells (ATCC) were cultured in DMEM complete medium containing $10 \%$ FBS and $100 \mathrm{U} / \mathrm{mL}$ of penicillin/streptomycin (Invitrogen). The cells were seeded at $5 \times 10^{4}$ per well in a standard 6-well plate and cultured before $70-80 \%$ confluency was reached. RFP-containing gelatin $(1 \mathrm{mg} \mathrm{RFP} / \mathrm{mL}) / \mathrm{TPB}$ composites were sterilized after exposed under UV-light for $30 \mathrm{~min}$ and transferred into cell strainers $(40 \mu \mathrm{m}$ mesh size, $\mathrm{BD})$. Next, the strainers 
were put in each well containing $2 \mathrm{~mL}$ of the medium. The composites and cells were coincubated at $37^{\circ} \mathrm{C}$ in a humidified atmosphere containing $5 \% \mathrm{CO}_{2}$. Cell viability was determined using Alamar Blue assay. Briefly, after $48 \mathrm{~h}$ of coculture, the cells were washed with fresh PBS and $2 \mathrm{~mL}$ of $1 \times$ Alamar Blue-DMEM medium was added to each well. After $4 \mathrm{~h}$ of incubation, $100 \mu \mathrm{L}$ of the medium in each well was transferred to a 96-well plate for reading. Absorbance intensities were recorded using a plate reader at wavelength of $570 \mathrm{~nm}$. Cell viability was calculated according to the following equation: cell viability $(\%)=\left(\mathrm{OD}_{\text {sample }}-\right.$ $\left.\mathrm{OD}_{0}\right) /\left(\mathrm{OD}_{\text {control }}-\mathrm{OD}_{0}\right) \times 100$, wherein $\mathrm{OD}_{\text {sample }}, \mathrm{OD}_{\text {control }}$, and $\mathrm{OD}_{0}$ represent the absorbance density of the medium with the composite, the medium without the composite, and $1 \times$ Alarm Blue-DMEM medium as a blank, respectively. Cells cultured without composite as a control were taken as $100 \%$ cell viability. All tests were performed in triplicate.

2.7. Surgical Procedures. Eighty-four young New Zealand rabbits (provided by Shanghai Second Military Medical University, Laboratory Animal Center, male or female) were randomly divided into 3 groups $(n=3)$. After the rabbits were anesthetized by injecting $3 \%$ Nembutal $(30 \mathrm{mg} / \mathrm{kg})$ via ear vein, the lateral femoral cortex and the center bottom part of femur were exposed. A $0.5 \mathrm{~cm}$ groove away from the articular surface of lateral femoral cortex was created by an osteotome ( $1.5 \mathrm{~cm}$ in length and $0.8 \mathrm{~cm}$ in width) [8]. RFPcontaining gelatin/TPB composite coated by PLA and TPB alone as the blank control group were, respectively, weighed and then embedded into the groove. A picture was taken to explain the surgery procedure in Figures 6(a) and 6(b). Finally, the muscle and skin were closed. All the rabbits were monitored after surgery. To observe neotissue formation, CT scanning (Phillips) was conducted at regular time intervals, that is, 4 and 8 week after the operation.

2.8. Histological Staining. The samples were decalcified in $10 \%$ formic acid, dehydrated in a gradient ethanol series, mounted in paraffin, sectioned $4 \mathrm{~mm}$ thick. To observe tissue formation, the sections were stained via haematoxylin-basic fuchsin-picric acid (HBFP) $[17,18]$. Briefly, deparaffinized sections were placed in a basic fuchsion-staining solution consisting of acetate buffer (pH 5.0) $50 \mathrm{mM}$ sodium tartrate, basic fuchsin (Sigma), and $0.5 \mathrm{mg} / \mathrm{mL}$ picric acid (Sigma) and incubated at $37^{\circ} \mathrm{C}$ for $5-10 \mathrm{~min}$. After the solution was removed by washing, sections were counterstained with hematoxylin and observed under light microscopy.

\section{Results}

3.1. Preparation and Characterization of Tricalcium Phosphate Bioceramics (TPB) Composites Loaded with Rifampicin(RFP-) Containing Gelatin Hydrogel. In this study, RFPcontaining gelatin solution was filled into microporous TPB scaffold by sufficient diffusion of the solution into microporous interior. RFP was then encapsulated inside the scaffold after in situ crosslinking of gelatin with calcium ions, thereby yielding a RFP-containing gelatin hydrogel/TPB binary composite (Scheme 1). In this way, different RFP or gelatin concentrations could be applied to give a group of binary composites. Figure 1 shows an imaging of the composite interior observed under scanning electronic microscopy. It was revealed that, compared with original TPB scaffold, additional substrate was found in porous region of RFPloaded gelatin/TPB composite, implying that gelatin was successfully loaded into the TPB-based composite.

3.2. In Vitro RFP Release from RFP-Loaded Gelatin/TPB Composites. To examine the possibility of gelatin hydrogel/TPB composites serving as drug delivery system, RFP-loaded composites were incubated in PBS buffer at $\mathrm{pH} 7.4$ and $37^{\circ} \mathrm{C}$. The released RFP was determined by UV/Vis spectrum. Figure 2 shows the release profiles of the composites that are prepared by using different concentrations of gelatin solution containing the same RFP concentration of $1 \mathrm{mg} / \mathrm{mL}$. In general, these composites revealed a burst and sustained release profile. Moreover, a slower release rate was obtained for those composites with higher concentrations of gelatin solution. For example, almost $90 \%$ of loaded RFP was already released out from the composite with $0.25 \mathrm{wt} \%$ and $0.5 \mathrm{wt} \%$ gelatin within $6 \mathrm{~h}$. However, at the same time, only $60 \%$ of the RFP was released from the composite with $1 \mathrm{wt} \%$ gelatin. For these gelatin hydrogel/TPB composites, their entrapment efficiency (EE) of RFP was determined as a function of RFP concentration in $1 \mathrm{wt} \%$ gelatin solution. As shown in Figure 3, the EE was decreased from about $60 \%$ to $20 \%$ with increasing RFP concentrations from 0.25 to $2.5 \mathrm{mg} / \mathrm{mL}$. However, the loading efficiency (LE) of RFP for these composites was increased from approximately $2 \%$ to $5 \%$ with increasing RFP concentrations. Overall, an optimal RFP concentration appeared to be $1 \mathrm{mg} / \mathrm{mL}$ so as to obtain moderate EE and LE. The binary TPB composite, prepared from $1 \mathrm{mg} / \mathrm{mL}$ of RFP in $1 \mathrm{wt} \%$ of gelatin solution, was thus used for further studies.

3.3. In Vitro RFP Release from Biodegradable Polymer-Coated, Gelatin Hydrogel/TPB Composites. On the basis of aforementioned results, RFP-containing gelatin hydrogel/TPB binary composites were further coated with a biocompatible, biodegradable polymer, that is, PLA, PLGA, and PCL, yielding ternary composites consisting of biodegradable polymer outside and RFP-loaded gelatin hydrogel inside (Scheme 1). These composites could be readily obtained by simply immersing RFP-loaded gelatin/TPB composite in the dichloromethane solution of these polymers and subsequent evaporation of the dichloromethane solvent. Figure 4 shows in vitro RFP release profiles of the composites under physiological conditions. These ternary composites could guild a sustained RFP release within a long period of time more than 20 days. It appears that biodegradable polymer has a direct effect on the release rate. For example, the composites coated by PLA and PLGA revealed a similar RFP release rate, but slower release rate as compared to PCLcoated composite. 

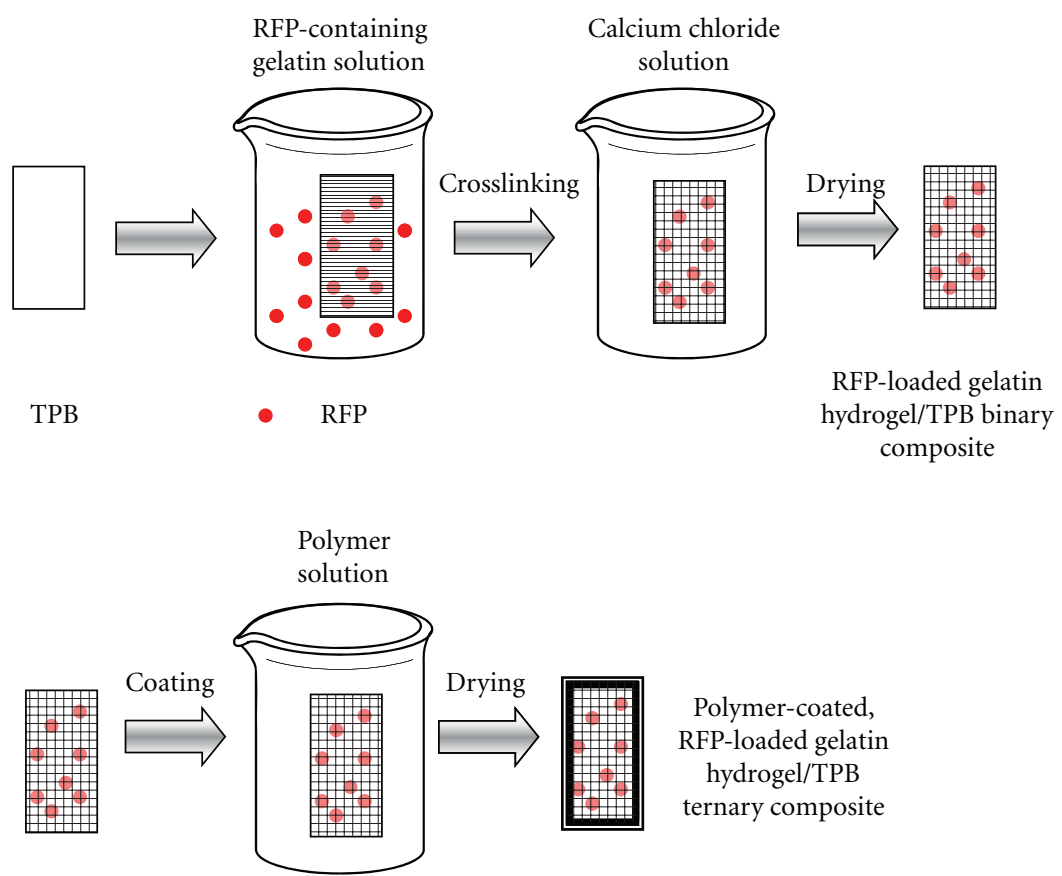

Scheme 1: The schematic illustration on the preparation of gelatin hydrogel/TPB binary composite loaded with rifampicin (RFP) and its ternary composite coated with a polymer (TPB: tricalcium phosphate bioceramics).

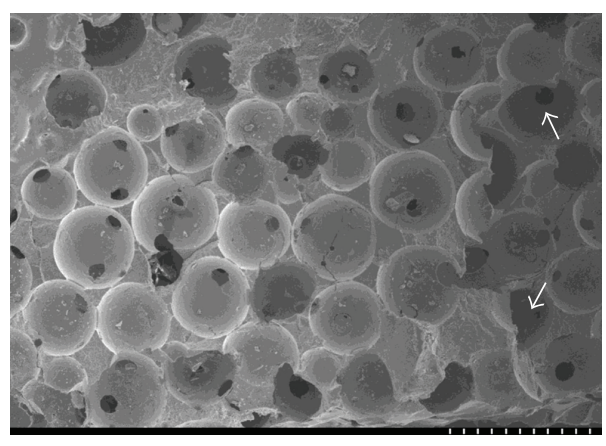

(a)

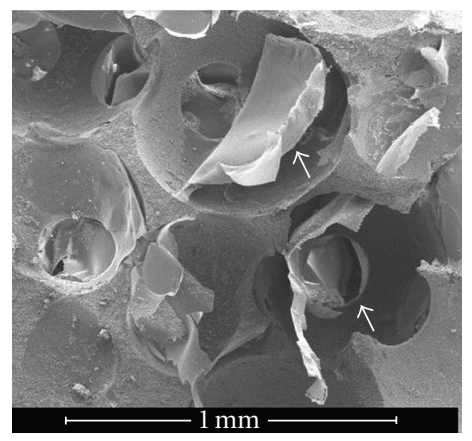

(b)

FIgURE 1: SEM images of the sections: (a) tricalcium phosphate bioceramics (TPB) and (b) gelatin hydrogel-loaded TPB.

3.4. In Vitro and In Vivo Biocompatibility of TPB-Based Composites. The cytobiocompatibility of RFP-loaded gelatin hydrogel/TPB ternary composites was evaluated against MC$3 \mathrm{~T} 3$ cells. The composite in a cell strainer was incubated with the cells for 2 days and cell survival was then determined by an Alamar Blue assay. The data in Figure 5 showed that the binary composite generally displayed low cytotoxicity (cell viability $>95 \%$ ). As a negative control, RFP alone led to adverse cytotoxicity at a high concentration of $40 \mu \mathrm{g} / \mathrm{mL}$, with about $85 \%$ cell viability.

To ascertain whether polymer coated, RFT-containing gelatin/TBP composites can be applied for tissue regeneration, the composite with PLA (Figure 6(a)), as a typical example, was implanted into the groove in a rabbit model (Figure 6(b)). In histological section from the defect sites after 4 weeks' operation, the composite was gradually decomposed and being incorporated with surrounding tissue (Figure 6(c)). A close visualization in Figure 6(e) showed that new collagen fiber tissue (in red) and muscle cells (in purple) could be found. After 8 weeks' operation, the residual composite became less and formed tissue was found in good align with surrounding bond tissue (Figure 6(d)). Besides this, muscle fibrous tissue was also seen in the lesion in the experimental group (Figure 6(f)). X-ray scanning further showed that, with operation time form 4 to 8 weeks, the lesion site displayed new tissue formation occupying in the groove from the experimental group, when compared to the blank control group (Figure 7).

\section{Discussion}

The treatment of osteoarticular tuberculosis by systemic drug administration has encountered a few issues such 


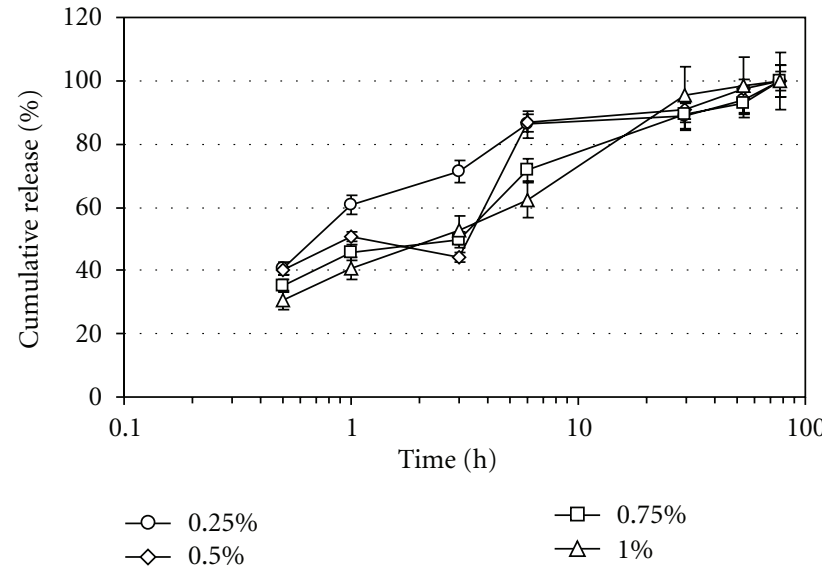

Figure 2: Delivery profiles of rifampicin (RFP) from $1 \mathrm{wt} \%$ RFP-loaded, gelatin hydrogel/TPB composite systems: the average cumulative percentage of RFP as a function of time, wherein different concentration of gelatin solution is applied. Error bars represent means \pm standard deviation $(n=3)$ for each formulation.

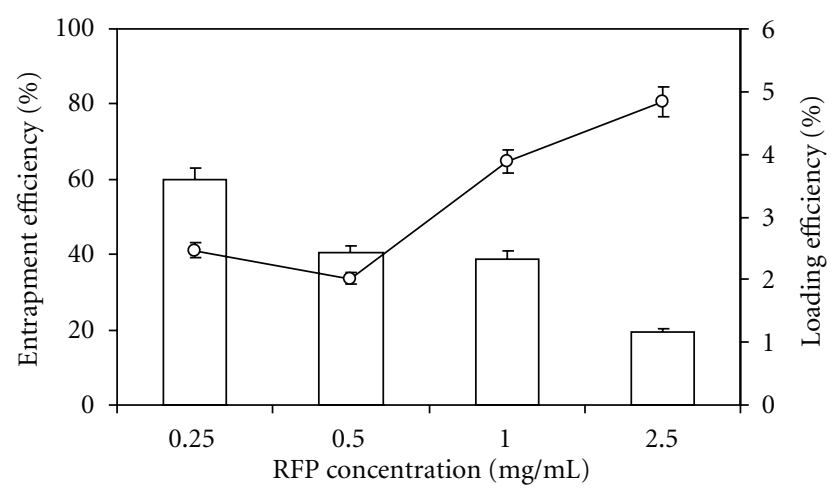

FIGURE 3: Entrapment efficiency (bar) and loading efficiency (line) of RFP as a function of the RFP concentration towards RFP-loaded, $1 \mathrm{wt} \%$-gelatin hydrogel/TPB composites.

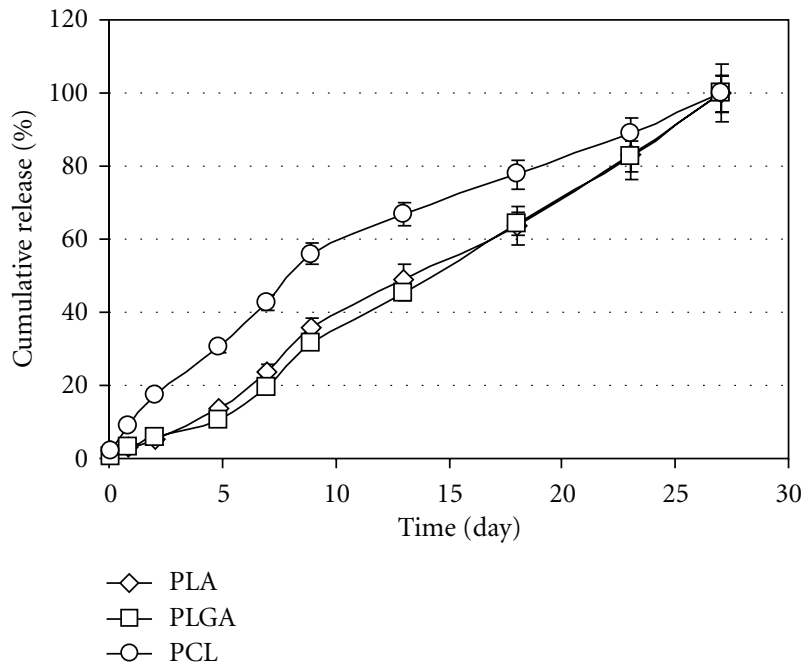

Figure 4: Cumulative release profiles of gelatin hydrogel/TPBbased ternary systems, loaded with RFP and coated by a biodegradable polymer such as PLA, PLGA, and PCL.

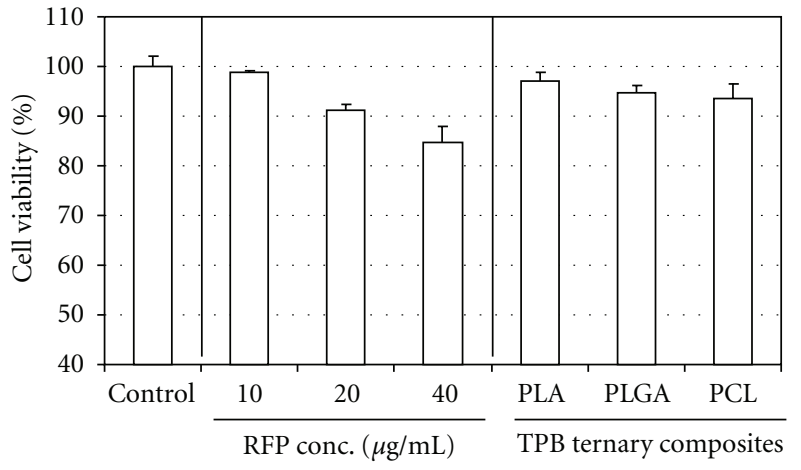

Figure 5: Cytotoxicity evaluation of RFP-loaded, biodegradable polymer-coated TPB-based ternary composites against MC-3T3 cells. As a control, cytotoxicity effect of RFP alone on MC-3T3 cells was also evaluated.

as serious side effect induced by multiple antitubercular drugs, long-term burden suffered from repeated administration, unsatisfied therapeutic efficacy due to low drug concentration at the osseous lesion [1]. In this study, we have fabricated an implantable composite as a drug delivery system for local osteoarticular tuberculosis therapy and meanwhile as a bioscaffold for regeneration of defect tissue. This composite system, consisting of gelatin hydrogel inside and biodegradable polymer outside, possesses four types of properties that are important for the treatment of osteoarticular tuberculosis. First, an appropriate dose of antitubercular drug can be loaded into gelatin hydrogelcontaining composites to maintain a local drug level above a minimal inhibitory concentration (Figure 2). Second, by using biodegradable polymer, the release duration of RFP could be as long as more than 20 days (Figure 3 ). Third, the RFP release profile from the system is sustained without a burst release. Fourth, the system is based on biocompatible materials and thus is very suited for tissue regeneration (Figures 5 and 6). These properties render the ternary composite system highly efficient for the sterilization of tubercle bacillus in osteoarticular site.

Long release duration of RFP from the gelatin hydrogel/TPB composite is highly desired to provide a long-term, sustained drug level for killing tubercle bacillus in defect sites. The concentration of gelatin reveals a direct effect on the duration time. The higher the gelatin solution, the longer the duration time of RFP (Figure 2). Obviously, this is most likely due to a higher crosslinking degree when applying a higher concentration of gelatin solution. For example, the longest release duration of about $80 \mathrm{~h}$ was obtained for the TPB composite containing $1 \mathrm{wt} \%$ gelatin. However, this duration time is relatively short. It is hard to prepare gelatin solution at a higher concentration above $1 \mathrm{wt} \%$ due to limited gelatin solubility. Thus, we further decorate gelatin hydrogel/TPB binary composite with a biodegradable polymer. As expected, longer release duration time more than 20 days was obtained without apparent burst release (Figure 4$)$. This duration time ( $\sim 26$ days) is comparable with that of the system from $\mathrm{TPB} /$ mesoporous silica nanoparticles 


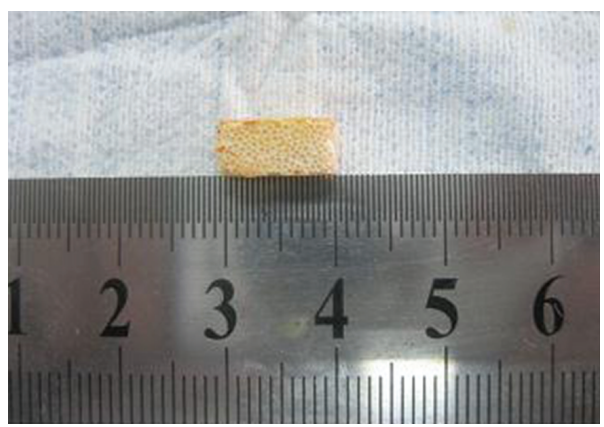

(a)

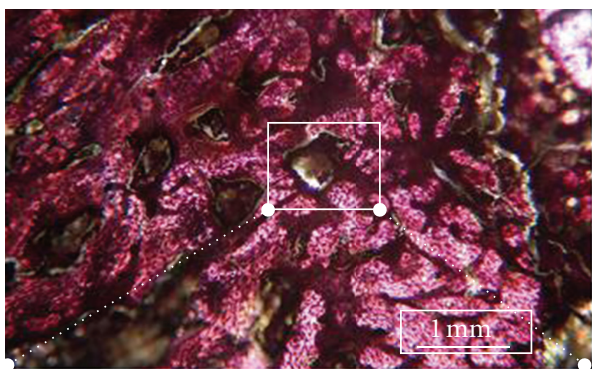

(c)

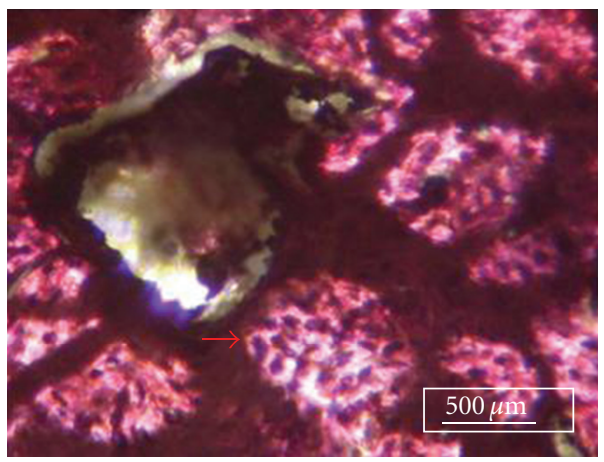

(e)

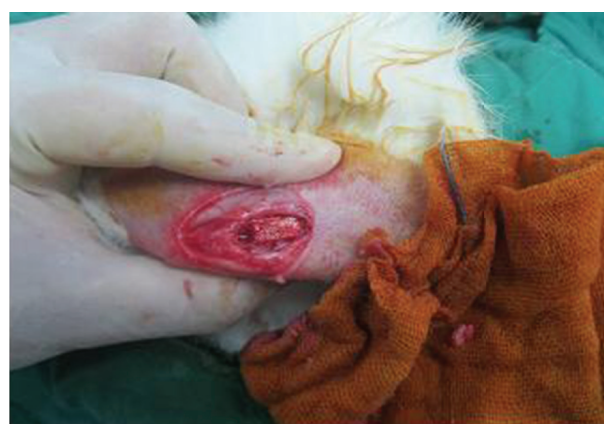

(b)

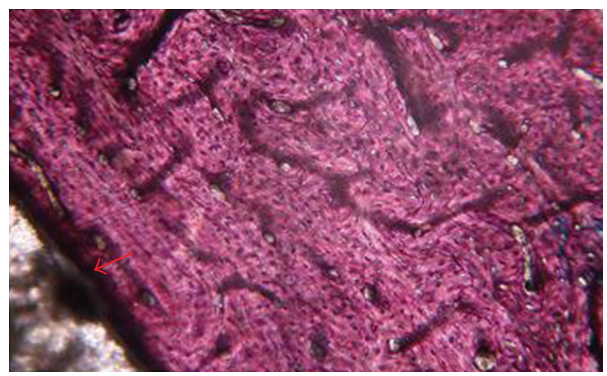

(d)

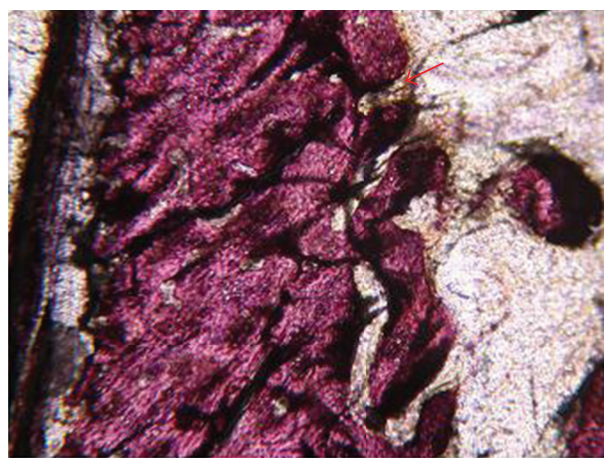

(f)

Figure 6: (a) A typical picture of RFP-loaded, PLA-coated gelatin/TPB ternary composite; (b) operation on the implantation of TPB ternary composite into the groove in a rabbit model; (c) deep observation of tissue section of implanted TPB ternary composite after 4-week operation; (d) tissue section of implanted TPB ternary composite after 8-week operation; a more close visualization of tissue section of implanted TPB ternary composite after 4-week operation (e) and 8-week operation (f).

in the previous report ( $\sim 32$ days) [8]. Notably, these biodegradable polymers are well known to be biocompatible and have no adverse effect on tissue regeneration after the polymer-based TPB ternary composites are implanted in the osteoarticular defect site.

Tricalcium phosphate bioceramics (TPB) has been widely studied as a bioscaffold for tissue engineering due to its high mechanical strength and good biocompatibility [11]. Besides, TPB possesses high affinity for bone tissue and also a significant ability to adsorb organic substances which are highly required for tissue regeneration in vivo [19]. As such, TPB composites integrated with biocompatible gelatin hydrogel and coated by PLA reveal good biocompatibility in vivo. In a rabbit model, neotissue formation was observed after implantation of PLA-coated TPB-based composite into a groove site (Figure 7), implying that the RFP released locally from the composite has no serious adverse effect on tissue regeneration. Therefore, the data confirm that, through a simple modification approach, TPB-based ternary system can be rationally designed for sustained antitubercular drug delivery and tissue regeneration.

\section{Conclusions}

We have demonstrated that a novel TPB ternary composite system can be prepared that is loaded with gelatin hydrogel inside and coated by a biodegradable polymer outside. These composites can be applied for sustained release of antitubercular drug rifampicin within a long period over 20 days. Besides, these composites display good biocompatibility in vitro against MC-3T3 cells and can be used for tissue regeneration in vivo in a rabbit model. The results of this 


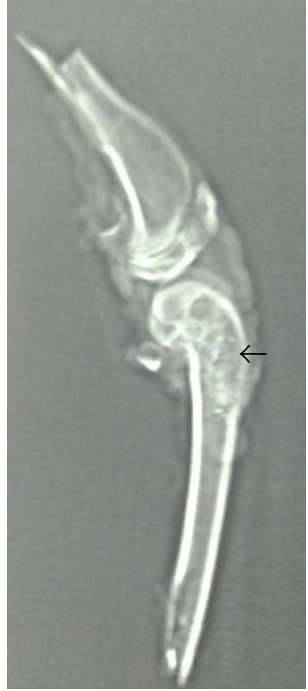

(a)

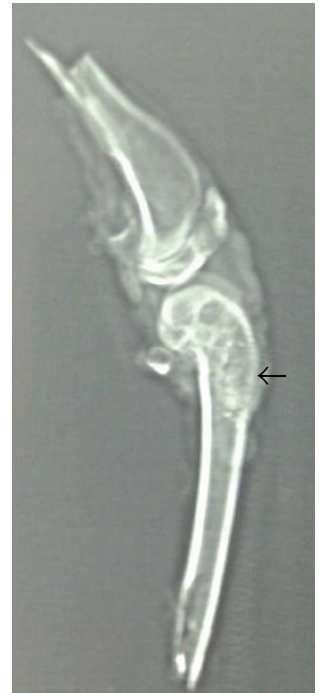

(b)

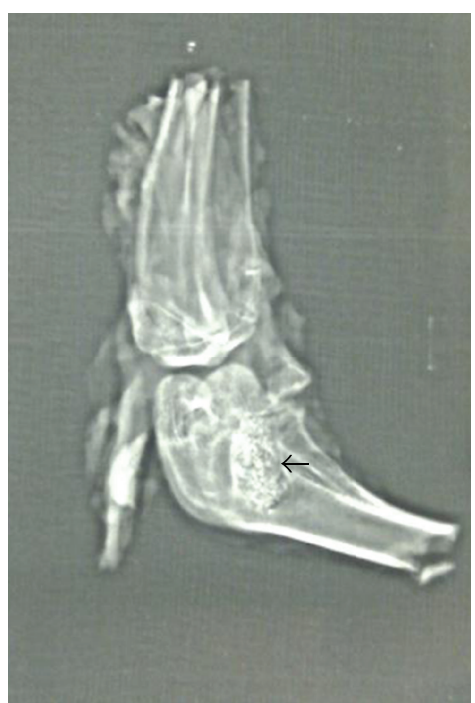

(c)

Figure 7: CT scanning to visualize new tissue formation in the groove at week 4 (b) and week 8 (c), after the implantation of PLA-coated, RFP-loaded gelatin hydrogel/TPB ternary composite. As a blank control (a), TPB alone was applied in the implantation operation for visualization at week 4 .

study suggest that the ternary composite system is promising for osteoarticular tuberculosis therapy.

\section{Acknowledgment}

This work was financially supported by the Shanghai Nanometer Special Project (no. 1052nm03203 and no. $11 \mathrm{~nm} 050420011)$.

\section{References}

[1] C. Lawlor, C. Kelly, S. O’Leary et al., "Cellular targeting and trafficking of drug delivery systems for the prevention and treatment of MTb," Tuberculosis, vol. 91, no. 1, pp. 93-97, 2011.

[2] A. Gürsoy, E. Kut, and S. Özkirimli, "Co-encapsulation of isoniazid and rifampicin in liposomes and characterization of liposomes by derivative spectroscopy," International Journal of Pharmaceutics, vol. 271, no. 1-2, pp. 115-123, 2004.

[3] S. Agal, R. Baijal, S. Pramanik et al., "Monitoring and management of antituberculosis drug induced hepatotoxicity," Journal of Gastroenterology and Hepatology, vol. 20, no. 11, pp. 1745-1752, 2005.

[4] W. W. Yew and C. C. Leung, "Antituberculosis drugs and hepatotoxicity," Respirology, vol. 11, no. 6, pp. 699-707, 2006.

[5] M. Dutt and G. K. Khuller, "Liposomes and PLG microparticles as sustained release antitubercular drug carriers-an in vitro-in vivo study," International Journal of Antimicrobial Agents, vol. 18, no. 3, pp. 245-252, 2001.

[6] K. Hirota, T. Hasegawa, T. Nakajima et al., "Delivery of rifampicin-PLGA microspheres into alveolar macrophages is promising for treatment of tuberculosis," Journal of Controlled Release, vol. 142, no. 3, pp. 339-346, 2010.

[7] T. Onoshita, Y. Shimizu, N. Yamaya et al., "The behavior of PLGA microspheres containing rifampicin in alveolar macrophages," Colloids and Surfaces B, vol. 76, no. 1, pp. 151$157,2010$.

[8] M. Zhu, H. Wang, J. Liu et al., "A mesoporous silica nanoparticulate/ $\beta$-TCP/BG composite drug delivery system for osteoarticular tuberculosis therapy," Biomaterials, vol. 32, no. 7, pp. 1986-1995, 2011.

[9] J. Lu, M. Liong, Z. Li, J. I. Zink, and F. Tamanoi, "Biocompatibility, biodistribution, and drug-delivery efficiency of mesoporous silica nanoparticles for cancer therapy in animals," Small, vol. 6, no. 16, pp. 1794-1805, 2010.

[10] S. Haldar, P. Ghosh, and A. Ghosh, "Tuberculous arthritisthe challenges and opportunities: observations from a tertiary center," Indian Journal of Rheumatology, vol. 6, no. 1, pp. 62$68,2011$.

[11] R. G. Carrodeguas and S. De Aza, " $\alpha$-Tricalcium phosphate: synthesis, properties and biomedical applications," Acta Biomaterialia, vol. 7, pp. 3536-3546, 2011.

[12] J. Ren, P. Zhao, W. Liu, and Q. Wu, "Preparation, mechanical, and thermal properties of biodegradable polyesters/poly(Lactic Acid) blends," Journal of Nanomaterials, vol. 2010, Article ID 287082, 8 pages, 2010.

[13] P. Zhao, W. Qin-Feng, Q. Zhong, Z. Nai-Wen, and J. Ren, "Microstructure and mechanism study of polylactide obtained by the copolymerization of l-lactide and d,l-lactide," Journal of Applied Polymer Science, vol. 115, no. 5, pp. 2955-2961, 2010.

[14] C. Lin, P. Zhao, F. Li, F. Guo, Z. Li, and X. Wen, "Thermosensitive in situ-forming dextran-pluronic hydrogels through Michael addition," Materials Science and Engineering C, vol. 30, no. 8, pp. 1236-1244, 2010.

[15] C. Lin and J. F. J. Engbersen, "PEGylated bioreducible poly(amido amine)s for non-viral gene delivery," Materials Science \& Engineering C-Materials for Biological Applications, vol. 31, no. 7, pp. 1330-1337, 2011.

[16] C. Lin and J. P. Ge, "Multifunctional polyethylenimineconjugated superparamagnetic nanoparticles for drug delivery and imaging," Journal of Controlled Release, vol. 152, supplement 1, pp. E58-E60, 2011. 
[17] A. T. Scherer and A. T. Masi, "Technical aspects of the Haematoxylin Basic Fuchsin picric acid (HBFP) stain applied to skeletal muscle," Histochemical Journal, vol. 7, no. 4, pp. 335-341, 1975.

[18] W. Xia, P. Wang, C. Lin et al., "Bioreducible polyethyleniminedelivered siRNA targeting human telomerase reverse transcriptase inhibits HepG2 cell growth in vitro and in vivo," Journal of Controlled Release, vol. 157, no. 3, pp. 427-436, 2012.

[19] T. Kawasaki, S. Takahashi, and K. Ikeda, "Hydroxyapatite highperformance liquid chromatography: column performance for proteins," European Journal of Biochemistry, vol. 152, no. 2, pp. 361-371, 1985. 

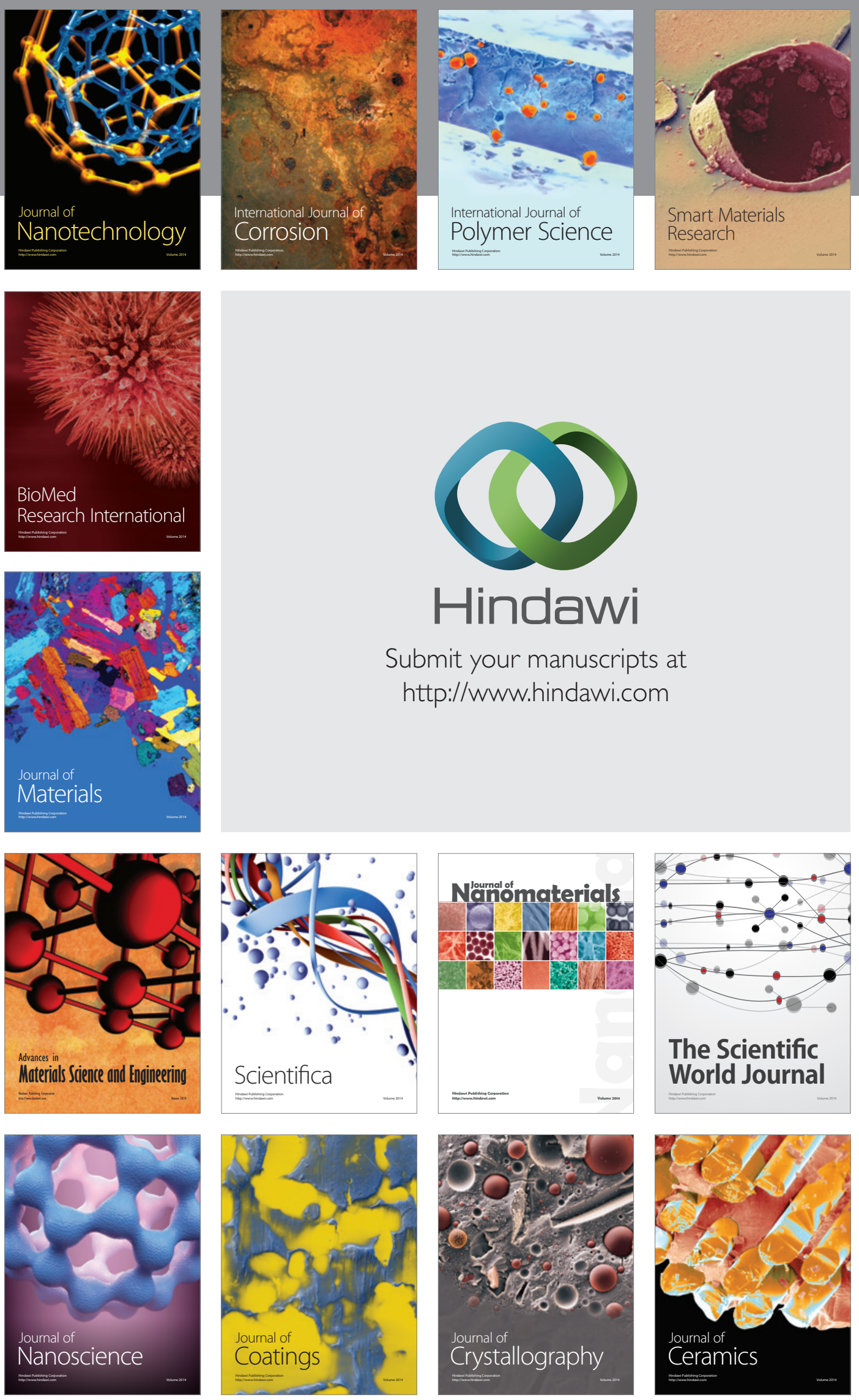

The Scientific World Journal

Submit your manuscripts at

http://www.hindawi.com

\section{World Journal}

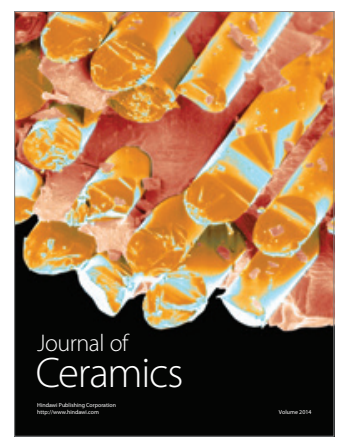

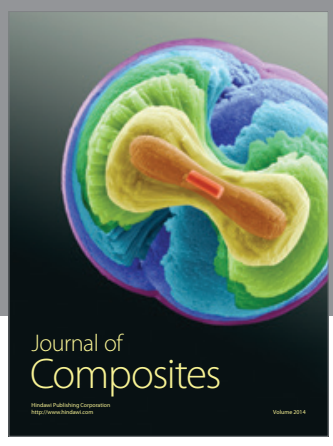
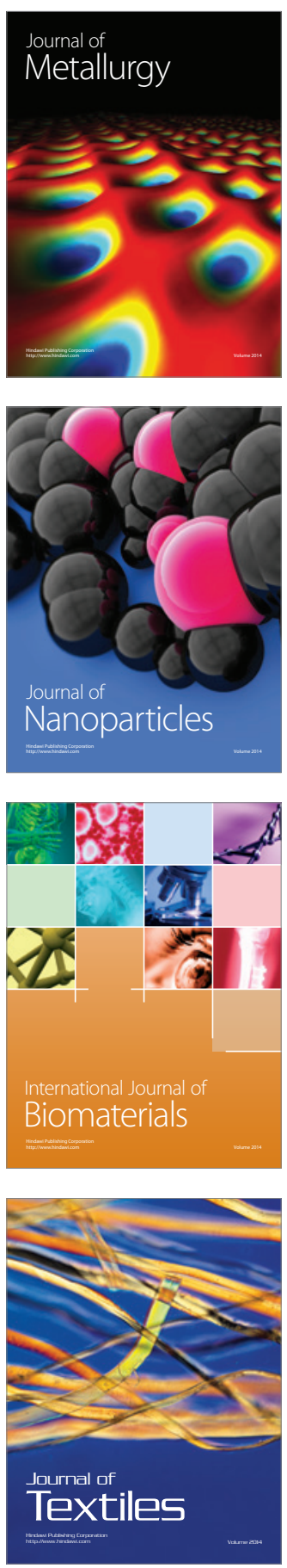